\title{
Strontium ranelate reduces cartilage degeneration and subchondral bone remodeling in rat osteoarthritis model
}

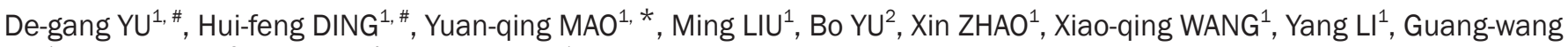
$\mathrm{LIU}^{1}$, Shao-bo NIE ${ }^{3}$, Shen $\mathrm{LIU}^{4}$, Zhen-an ZHU ${ }^{1}$

\begin{abstract}
${ }^{1}$ Department of Orthopedics, Shanghai Ninth People's Hospital, Shanghai Jiao Tong University, School of Medicine, Shanghai 200011, China; ${ }^{2}$ Department of Orthopedics, Affiliated Hospital of Shandong University of Traditional Chinese Medicine, Ji-nan 250014, China;

${ }^{3}$ Department of Orthopedics, PLA General Hospital, Beijing 100853, China; ${ }^{4}$ Department of Orthopedics, Shanghai Sixth People's Hospital, Shanghai Jiao Tong University, Shanghai 200233, China
\end{abstract}

\begin{abstract}
Aim: To investigate whether strontium ranelate (SR), a new antiosteoporotic agent, could attenuate cartilage degeneration and subchondral bone remodeling in osteoarthritis (OA).

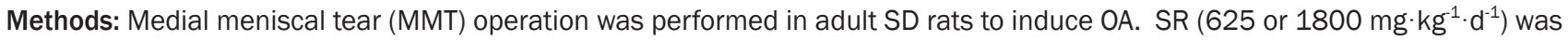
administered via gavage for 3 or 6 weeks. After the animals were sacrificed, articular cartilage degeneration was evaluated using toluidine blue 0 staining, SOX9 immunohistochemistry and TUNEL assay. The changes in microarchitecture indices and tissue mineral density (TMD), chemical composition (mineral-to-collagen ratio), and intrinsic mechanical properties of the subchondral bones were measured using micro-CT scanning, confocal Raman microspectroscopy and nanoindentation testing, respectively.

Results: The high-dose SR significantly attenuated cartilage matrix and chondrocyte loss at 6 weeks, and decreased chondrocyte apoptosis, improved the expression of SOX9, a critical transcription factor responsible for the expression of anabolic genes type II collagen and aggrecan, at both 3 and 6 weeks. Meanwhile, the high-dose SR also significantly attenuated the subchondral bone remodeling at both 3 and 6 weeks, as shown by the improved microarchitecture indices, TMD, mineral-to-collagen ratio and intrinsic mechanical properties. In contrast, the low-dose SR did not significantly change all the detection indices of cartilage and bone at both 3 and 6 weeks. Conclusion: The high-dose SR treatment can reduce articular cartilage degeneration and subchondral bone remodeling in the rat MMT model of OA.
\end{abstract}

Keywords: osteoarthritis; antiosteoporotic agent; strontium ranelate; medial meniscal tear; articular cartilage; subchondral bone; SOX9 Acta Pharmacologica Sinica (2013) 34: 393-402; doi: 10.1038/aps.2012.167; published online 21 Jan 2013

\section{Introduction}

Osteoarthritis (OA), a leading cause of morbidity in the aging population ${ }^{[1]}$, is characterized by progressive loss of articular cartilage, subchondral bone lesions and a slight synovial reaction ${ }^{[2]}$. The search for effective agents attenuating OA progression has been an ongoing and difficult process. For decades, articular cartilage has been the main target of major basic research and pharmaceutical therapies, but recent data indicate a key role of the subchondral bone tissue in the pathogenesis of $\mathrm{OA}^{[3,4]}$. Under physiological conditions, subchon-

\footnotetext{
"The two authors contributed equally to this work.

${ }^{*}$ To whom correspondence should be addressed.

E-mail yuanqingmao@163.com

Received 2012-06-24 Accepted 2012-11-14
}

dral bone has been shown to exert important shock-absorbing functions, attenuating approximately $30 \%$ of the joint load, and to provide a mechanical base for articular cartilage in normal joints ${ }^{[5]}$. In addition, subchondral bone supplies nutrients to cartilage and facilitates the removal of metabolic waste products ${ }^{[6]}$. However, subchondral abnormalities occur during OA progression. Bone turnover, as the main biological determinant of bone mineralization, increases dramatically in the subchondral bone $e^{[3,7,8]}$, which results in disorganized bone architecture, decreased mineralization and weakened biomechanical properties ${ }^{[9-11]}$. Furthermore, osteoblasts and osteoclasts, the cellular effectors of the anabolic and catabolic processes of bone tissue, manifest abnormal functions in osteoarthritic subchondral bone ${ }^{[12-14]}$, while producing various cytokines, growth factors, prostaglandins and leukotrienes, 
which seep through the bone-cartilage interface, initiate a chondrocyte phenotype shift toward hypertrophic differentiation and promote cartilage degradation ${ }^{[15,16]}$. Taken together, the present data indicate that, biologically and mechanically, abnormal subchondral bone would influence the integrity of the overlying cartilage ${ }^{[17,18]}$.

Strontium ranelate (SR), a new antiosteoporotic agent ${ }^{[19,20]}$, is able to increase preosteoblast replication, osteoblast differentiation, collagen type I synthesis and bone matrix mineralization. Paralleling these anabolic effects, SR is able to inhibit osteoclast differentiation and activity and enhance osteoclast apoptosis. The overall effect is a rebalanced bone turnover in favor of improving bone geometry, trabecular bone morphology and intrinsic bone tissue quality, which globally results in enhanced bone strength. Moreover, SR could increase cartilage matrix formation by ionic stimulation of human knee chondrocyte anabolism in vitro ${ }^{[21]}$. A recent study found that SR could inhibit key factors that affect bone remodeling in human osteoarthritic subchondral bone osteoblasts in vitro ${ }^{[22]}$. Based on these pharmacological findings, SR would be expected to attenuate osteoarthritic joint degeneration.

The commonly used rat medial meniscal tear (MMT) model of OA has been well characterized in terms of chondropathy and subchondral bone remodeling ${ }^{[23,24]}$. Because this model exhibits dynamic subchondral bone alteration, the cartilageand bone-preserving activities of therapeutic agents can be evaluated in this model ${ }^{[24]}$. The present study, using the established rat MMT model, investigated whether SR treatment could attenuate articular cartilage degeneration and subchondral bone remodeling.

\section{Materials and methods}

Animals

Adult male Sprague Dawley rats (12 weeks old, weighing $289 \pm 13 \mathrm{~g}$ ) from Sino-British Sippr/BK Lab Animal Ltd (Shanghai, China) were used in the present study. The animals were group housed under a 12-h light/dark cycle with food and water available ad libitum. The animals received standard laboratory food, which contains $1.56 \%$ calcium, $0.8 \%$ phosphorus, and $800 \mathrm{IU} / \mathrm{kg}$ vitamin D. The Animal Care and Experiment Committee of the Shanghai Jiaotong University School of Medicine approved all experimental procedures.

\section{Induction of $\mathrm{OA}$}

The rats were anesthetized with $10 \%$ chloral hydrate in phosphate-buffered saline (PBS, $0.01 \mathrm{~mol} / \mathrm{L}$ ) after 1 week of acclimatization. The MMT model was induced as previously described $^{[23]}$. Briefly, the medial collateral ligament of the right knee was transected, and the medial meniscus was reflected proximally toward the femur and cut through at its narrowest point. For the controls (Sham), the wounds were closed after exposing the medial collateral ligament.

\section{Pharmaceutical treatment}

A total of 128 animals were randomly divided into four groups ( $n=32$ per group). Pharmaceutical intervention com- menced from the first postoperative day. SR (OSSEOR ${ }^{\circledR}$, Les Laboratoires Servier Industrie, France) prepared as a suspension in normal saline (NS) was administered daily by gavage $(6 \mathrm{~mL} / \mathrm{kg})$ at two doses: low dose, $625 \mathrm{mg} / \mathrm{kg}$ (SRL); and high dose, $1800 \mathrm{mg} / \mathrm{kg}(\mathrm{SRH})$. The same volume of NS was given to the Sham (Sham/NS) and MMT animals (MMT/NS). Sixteen rats from each group were sacrificed at 3 and 6 weeks following the induction of OA. The doses adopted were based on previous studies on osteoporosis ${ }^{[25,26]}$. During treatment, body weights were obtained weekly, and the drug dose was adjusted accordingly every week.

\section{Tissue preparation}

The entire knee joint was dissected and fixed in $4 \%$ paraformaldehyde for $48 \mathrm{~h}$. A total of 8 joints per group at each time point were decalcified in $10 \%$ EDTA for 3 weeks. Then, the joints were bisected along the collateral ligament in the frontal plane, and both sections were embedded in the same paraffin block. The samples were cut into $5 \mu \mathrm{m}$ sections and prepared for subsequent evaluation of cartilage degeneration, including toluidine blue $\mathrm{O}$ staining, TUNEL histochemistry, and SOX9 immunohistochemistry. Another 8 undecalcified joints were used for the evaluation of changes in the subchondral bone, including micro-CT imaging, confocal Raman microspectroscopy, and nanoindentation.

\section{Histological analysis}

On the basis of the OARSI recommendations for the histological assessment of osteoarthritis in the rat ${ }^{[24]}$, three sections from each knee at $200 \mathrm{~mm}$ steps were stained with toluidine blue $\mathrm{O}$ and subsequently evaluated for cartilage degeneration. Briefly, the sections were stained with $0.04 \% w / v$ toluidine blue O (Sigma, T0394) in $0.1 \mathrm{~mol} / \mathrm{L}$ sodium acetate $(\mathrm{pH} 4.0)$ for $10 \mathrm{~min}$, rinsed, air-dried and mounted.

According to OARSI recommendations ${ }^{[24]}$, cartilage degeneration was scored. The medial tibial plateau was divided into three regions of equal width and cartilage degeneration in each zone was scored 'none' to 'severe' (numerical values $0-5)$ using the criteria described in Table 1 . The original surface of the tissue was estimated. Then the $\%$ area of each zone that contains cartilage exhibiting loss of chondrocytes or loss of matrix was estimated and a score was assigned to that zone based on that percentage. The total cartilage degeneration score was calculated by adding the values obtained for each

Table 1. OARSI recommended cartilage degeneration score for rat.

\begin{tabular}{ll}
\hline Grade & \multicolumn{1}{c}{ Description } \\
\hline 0 & $\begin{array}{l}\text { No degeneration } \\
\text { Minimal degeneration; } 5 \%-10 \% \text { of the total projected cartilage } \\
\text { area affected by matrix or chondrocyte loss }\end{array}$ \\
& Mild degeneration; $11 \%-25 \%$ affected \\
3 & Moderate degeneration; $26 \%-50 \%$ affected \\
4 & Marked degeneration; $51 \%-75 \%$ affected \\
5 & Severe degeneration; greater than $75 \%$ affected
\end{tabular}


zone.

\section{TUNEL assay}

The TUNEL method was used to determine chondrocyte apoptosis in the knee joint cartilage. Three sections from each knee were processed for TUNEL histochemistry (Roche, In Situ Cell Death Detection Kit, Fluorescein, cat N 11684795910) according to the manufacturer's instructions and then counterstained with 4',6-diamidino-2-phenylindole (DAPI) to label the nuclei. Chondrocyte apoptosis was determined as the percentage of TUNEL-positive nuclei in the DAPI-labeled nuclei for each individual rat and averaged for each treatment group.

\section{SOX9 immunohistochemistry}

Three sections from each knee were selected for SOX9 immunohistochemistry. The deparaffinized and rehydrated sections were incubated with a trypsin antigen retrieval solution at $37^{\circ} \mathrm{C}$ for $15 \mathrm{~min}$ and subsequently with $3 \% \mathrm{H}_{2} \mathrm{O}_{2}$ at room temperature (RT) for 15 min to block endogenous peroxidases. After blocking with 10\% normal goat serum, the sections were incubated with rabbit anti-rat SOX9 (1:50, Santa Cruz Biotechnology, sc-20095) overnight at $4^{\circ} \mathrm{C}$. Next, the sections were incubated with goat anti-rabbit Alexa 594 (1:500, Molecular Probes, A11012) for $1 \mathrm{~h}$ at RT. Finally, the sections were counterstained with DAPI. The number of SOX9-positive chondrocytes was quantified.

All images were acquired at 100× magnification using a fluorescence microscope (Leica DM 4000B) with BioQuant OSTEO II software (BioQuant Image Analysis Corporation, Nashville, TN). The images were analyzed with Image-Pro Plus 6.0 (Media Cybernetics).

\section{Micro-CT imaging and analysis}

Structural alterations and the tissue mineral content in the subchondral bone were evaluated using micro-CT. The knee joints were scanned by micro-CT ( $\mu$ CT 80; SCANCO Medical AG) with an isotropic voxel resolution of $10 \mu \mathrm{m}$. As shown in our previous study ${ }^{[27]}$, a portion (2.0 $\mathrm{mm}$ ventrodorsal length) of the load-bearing region at the medial tibial plateau was obtained as a region of interest. The following parameters for the subchondral trabecular bone were calculated: TMD, bone volume fraction (BV/TV), trabecular thickness (Tb.Th), trabecular spacing (Tb.Sp), trabecular number (Tb.N), and connectivity density (Cnn.D).

\section{Confocal Raman microspectroscopy}

The chemical composition of the subchondral bone tissue (mineral-to-collagen ratio, $\mathrm{v}_{1}-\mathrm{PO}_{4}{ }^{3-} /$ proline) was characterized by confocal Raman microspectroscopy (Renishaw Inc, Hoffman Estates, IL, USA), as described previously ${ }^{[28,29]}$. After micro-CT scanning, joint samples were dehydrated in a graded alcohol series, embedded in methyl methacrylate (MMA) and sliced in the frontal plane using a Leica SP1600 saw microtome. The section surface was ground on successive grits of wet silicon carbide paper and polished by hand with $1 \mu \mathrm{m}$ alumina slurry, and 3 sections of $30 \mu \mathrm{m}$ thickness from each sample were obtained and analyzed. A 50× objective focused the laser (785 $\mathrm{nm}$ laser diode source) to a $3 \mu \mathrm{m}$ region within the medial tibial plateau, and the Raman scattered light was collected by a spectrograph with $0.5 \mathrm{~cm}^{-1}$ spectral resolution. The measured spectra consisted of three accumulations with an integration time of $10 \mathrm{~s}$ each. Using custom-developed software (Matlab, Mathworks, Natick, MA), the background fluorescence in the spectra was subtracted by a modified polynomial fitting algorithm ${ }^{[30]}$. Spectra were collected from 8 trabecular locations within each section, and $\mathrm{v}_{1}-\mathrm{PO}_{4}{ }^{3-} /$ proline was calculated as the raw $\mathrm{v}_{1}$ phosphate peak intensity $\left(\mathrm{v}_{1}-\mathrm{PO}_{4}{ }^{3-}, 962\right.$ $\mathrm{cm}^{-1}$ ) per proline peak intensity $\left(856 \mathrm{~cm}^{-1}\right)$.

\section{Nanoindentation testing}

The mechanical properties at the tissue level were quantified by nanoindentation as described previously ${ }^{[31]}$. Following the confocal Raman microspectroscopy, the medial tibial plateau of MMA-embedded knee sections were probed using a TriboIndenter (Hysitron Inc, Minneapolis, MN). A Berkovitch diamond tip (inclination angle $142.35^{\circ}$, radius $200 \mathrm{~nm}$ ) was pressed into the surface using a trapezoidal loading scheme as follows: 1) loading up to a nominal load of $1000 \mu \mathrm{N}$ at a rate of $200 \mu \mathrm{N} / \mathrm{s}$; 2) holding at $P_{\max }$ for $5 \mathrm{~s}$; and 3) unloading to zero load at a rate of $200 \mu \mathrm{N} / \mathrm{s}$. Thermal drift was corrected in the measurement software (Triboscan) by measuring the indenter drift for a maximum of $40 \mathrm{~s}$ before the start of every indent and applying this correction to the measured data. From the resulting force-displacement curve, the elastic modulus $\mathrm{E}_{\mathrm{r}}$ and hardness $\mathrm{H}$ of the tissue at the point of indention $(0.25 \mu \mathrm{m}$ resolution) was calculated following the method of Oliver and Pharr $^{[32]}$. Eight trabecular locations per section were chosen, and 4 indents for each location were tested and averaged per section.

\section{Statistical analysis}

All statistical analysis was performed by blinded authors who were unaware of the treatments given. The results are presented as the means \pm standard deviation (SD). Mann-Whitney non-parametric analyses were used to compare cartilage degeneration scores. Comparisons between groups for SOX9 immunohistochemistry, TUNEL histochemistry, micro-CT scanning, confocal Raman microspectroscopy and nanoindentation was performed with one-way analysis of variance using the LSD post-hoc test. $P$ values less than 0.05 were considered statistically significant.

\section{Results}

Animals

Treatment of MMT rats with the low dose of $625 \mathrm{mg} \cdot \mathrm{kg}^{-1} \cdot \mathrm{d}^{-1}$ or the high dose of $1800 \mathrm{mg} \cdot \mathrm{kg}^{-1} \cdot \mathrm{d}^{-1}$ of SR for 6 weeks was welltolerated and safe, with no significant or adverse effects on animal survival and body weight. The body weight of all animals increased throughout the course of the experiment, but SR showed no significant effect on the body weight gain. 
A
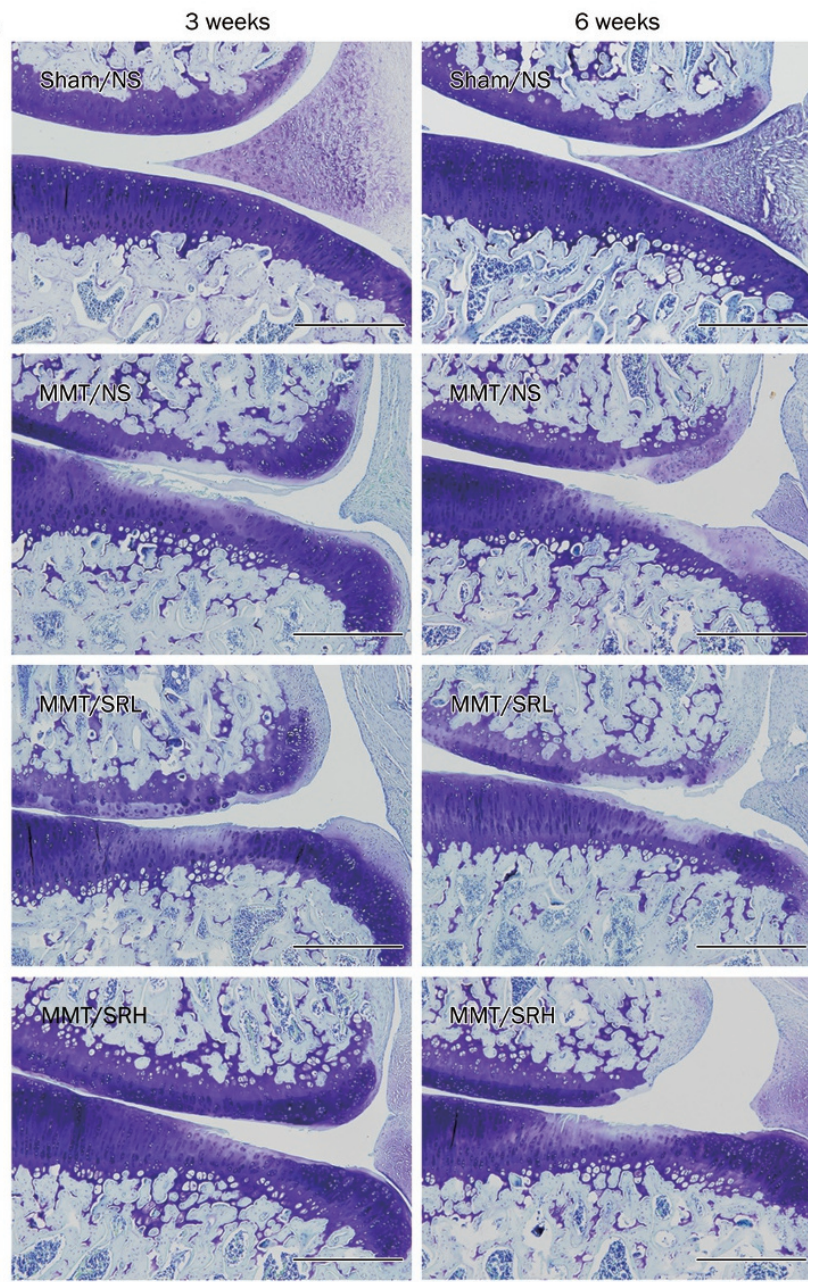

B

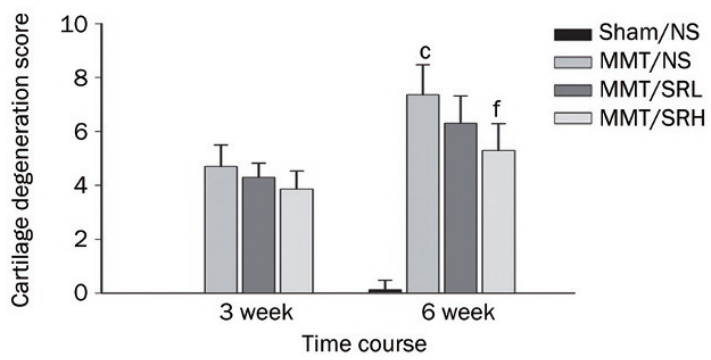

Figure 1. Effect of SR treatment on articular cartilage degeneration. Representative images with toluidine blue 0 staining. Images were acquired at $100 \times$ magnification. Bars $=500 \mu \mathrm{m}$. (B) Cartilage degeneration score. $n=8$ per group. Mean \pm SD. ${ }^{c} P<0.01$ vs the Sham/NS group. ${ }^{\text {f}} P<0.01$ vs MMT/NS group.

\section{Effect of SR treatment on articular cartilage degeneration}

As indicated by toluidine blue $O$ staining (Figure 1), the MMT induced articular cartilage degeneration on the medial tibia platform, whereas the Sham joints showed no obvious changes at both time points. At 3 weeks, the MMT/NS joints exhibited moderate cartilage degeneration; matrix and chondrocyte loss affected the middle to deep zone, and the cartilage degeneration score was $4.75 \pm 0.7$. At 6 weeks, severe cartilage degeneration occurred; matrix and chondrocyte loss deepened to the tidemark, and the cartilage degeneration

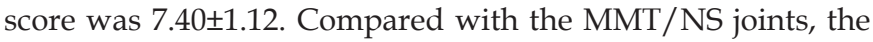
low-dose SR treatment showed no statistical improvement in cartilage degeneration both at 3 and 6 weeks, and corresponding cartilage degeneration scores were $4.30 \pm 0.53(P>0.05)$ and $6.31 \pm 1.00(P>0.05)$. Meanwhile, the high-dose SR treatment effectively attenuated the cartilage degeneration at 6 weeks, with the depth and width of the matrix and chondrocyte loss significantly reduced, and the cartilage degeneration score was $5.30 \pm 0.99(P<0.01)$ at 6 weeks.

\section{Effect of SR treatment on articular chondrocyte apoptosis}

Representative images of articular chondrocyte apoptosis as evaluated by TUNEL histochemistry are shown in Figure 2. The percentage of TUNEL-positive chondrocytes was significantly increased in the MMT/NS joints $(16.23 \% \pm 3.97 \%$ at 3 weeks and $21.17 \% \pm 3.45 \%$ at 6 weeks) compared with that in the Sham/NS joints $(1.77 \% \pm 0.64 \%$ at 3 weeks and $2.18 \% \pm 0.82 \%$ at 6 weeks). Compared with the MMT/NS joints, SR treatment at the low dose showed no significant reduction in chondrocyte apoptosis: the percentages of TUNEL-positive chondrocytes were $14.98 \% \pm 1.90 \%(P>0.05)$ at 3 weeks and $20.27 \% \pm 4.16 \%(P>0.05)$ at 6 weeks. However, SR treatment at the high dose caused significant improvements in chondrocyte apoptosis at both 3 and 6 weeks, with $7.44 \% \pm 1.96 \%(P<0.01)$ and $15.85 \% \pm 2.73 \%(P<0.01)$ TUNEL-positive chondrocytes, respectively.

\section{Effect of SR treatment on SOX9 expression in articular chondro- cytes}

As indicated by the immunofluorescence analysis (Figure 3), SOX9 was regularly expressed in the Sham/NS articular cartilage and reduced in the MMT/NS joints. However, compared with the MMT/NS joints, the expression of SOX9 was significantly improved by the high-dose SR treatment. Specifically, the number of SOX9-positive chondrocytes in SRH articular cartilage was $209.63 \pm 23.31(P<0.01)$ at 3 weeks and $163.75 \pm 20.80(P<0.01)$ at 6 weeks, compared with $173.75 \pm 26.07$ and $115.13 \pm 19.52$, respectively, in the MMT/NS joints. The SRL joints did not manifest a statistical improvement in the SOX9 expression at both 3 and 6 weeks, with 187.38 \pm 24.38 $(P>0.05)$ and 131.63 $\pm 22.22(P>0.05)$ SOX9-positive cells, respectively.

\section{Effect of SR treatment on subchondral microarchitecture and TMD}

The micro-CT scans and analysis (Figure 4) clearly demonstrated that the subchondral trabecular bone in the Sham/ NS groups was regularly aligned, but that obvious abnormal trabecular bone was present in the MMT/NS groups. In addition, TMD and BV/TV were decreased at 3 and 6 weeks, Tb.Th was thinned at 3 weeks and thickened at 6 weeks, Tb.Sp was broadened at 3 weeks and narrowed at 6 weeks, and Tb.N and Cnn.D were reduced at both 3 and 6 weeks in the MMT/ NS animals. These abnormal microstructure indices and TMD 
A
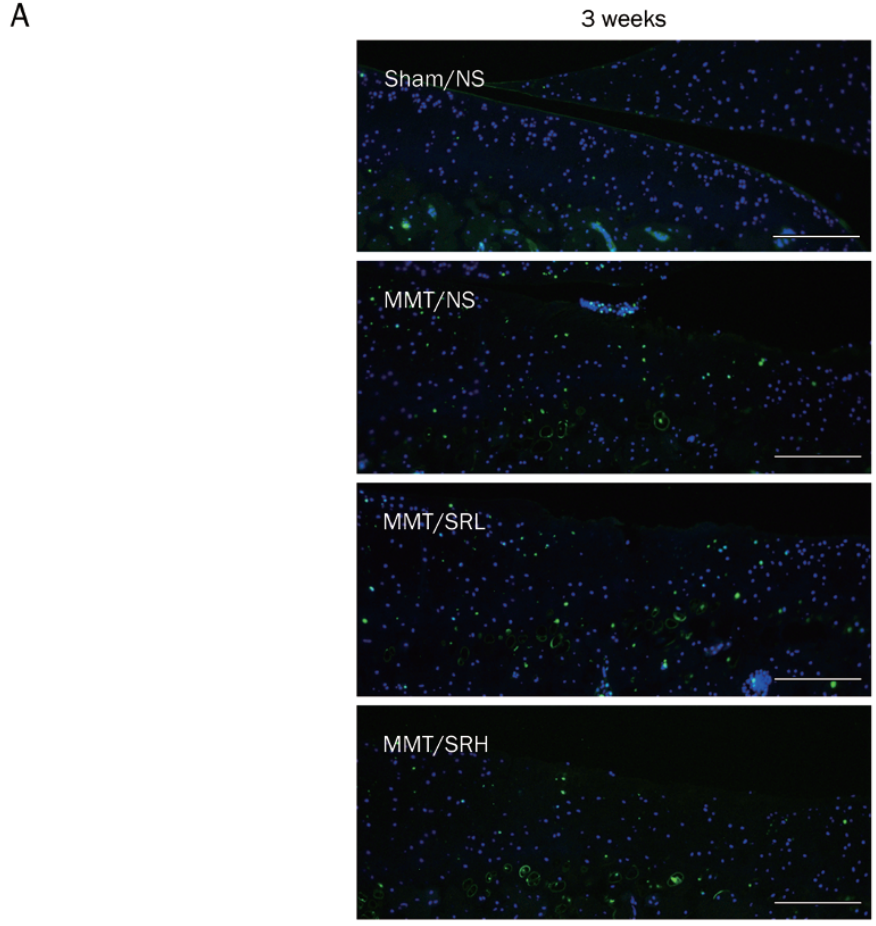
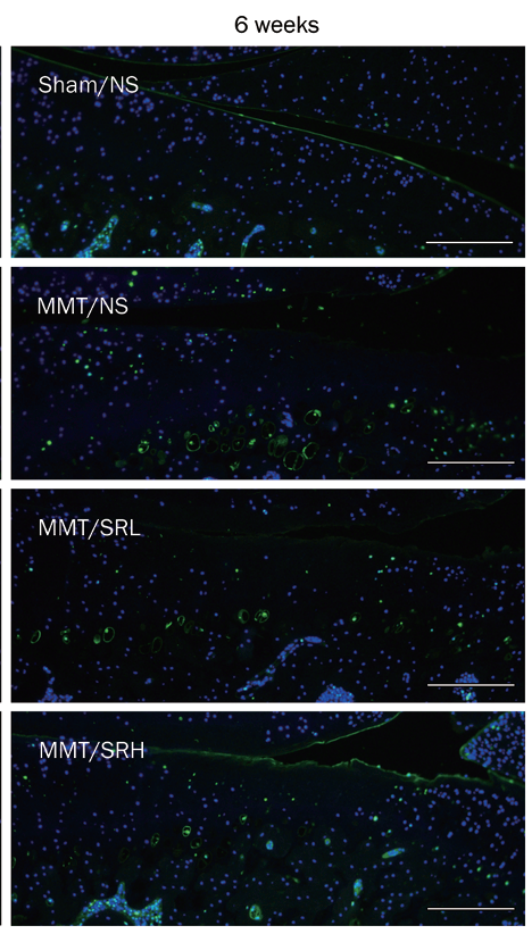

B

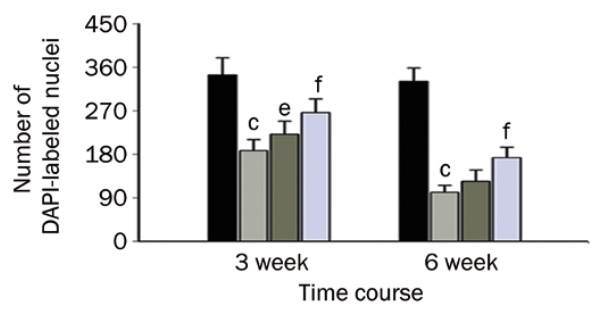

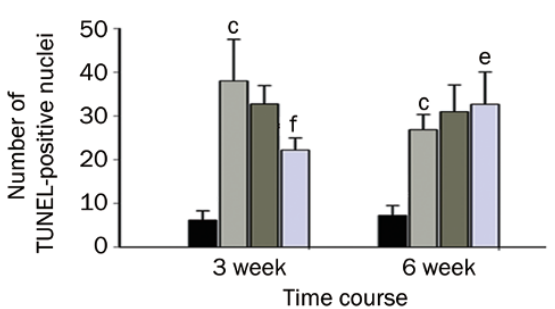

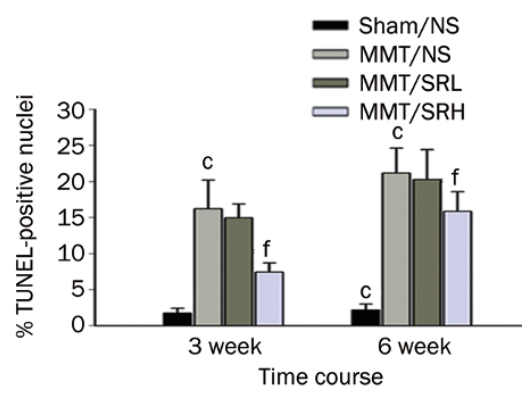

Figure 2. Effect of SR treatment on articular chondrocyte apoptosis. Representative images of chondrocyte apoptosis as evaluated by TUNEL histochemistry. Green indicates the TUNEL-positive chondrocytes. Blue indicates DAPI-labeled nuclei. The images were acquired at $\times 100$ magnification. Bars $=200 \mu \mathrm{m}$. (B) The percentage of TUNEL-positive chondrocytes out of the DAPI-labeled nuclei. $n=8$ per group. Mean \pm SD. ${ }^{c} P<0.01 \mathrm{vs}$ the Sham/NS group. ${ }^{\mathrm{e}} \mathrm{P}<0.05,{ }^{\mathrm{f}} \mathrm{P}<0.01$ vs MMT/NS group.

were significantly improved in the animals treated with SR at the high dose at both 3 and 6 weeks, whereas no significant improvement was observed in the animals treated with SR at the low dose, although the latter did show a tendency to improve.

\section{Effect of SR treatment on subchondral bone mineral-to-collagen ratio}

The mineral-to-collagen ratio $\left(\mathrm{v}_{1}-\mathrm{PO}_{4}^{3-} /\right.$ proline $)$ of the subchondral bone tissue calculated as $\mathrm{v}_{1}-\mathrm{PO}_{4}^{3-}$ peak intensity per proline peak intensity using confocal Raman microspectroscopy is shown in Figure 5. No significant difference in the mineral-to-collagen ratio among groups was observed at 3 weeks post-MMT operation, but at 6 weeks, the MMT/NS animals showed a reduction in $\mathrm{v}_{1}-\mathrm{PO}_{4}^{3-} /$ proline $(6.93 \pm 1.66)$ compared with the Sham/NS animals $(9.84 \pm 1.25, P<0.01)$. The reduced ratio was significantly improved by the high-dose SR treatment but not by the low-dose $(8.33 \pm 1.92, P>0.05$ for the
SRL animals and $9.01 \pm 1.43, P<0.05$ for the SRH animals).

\section{Effect of SR treatment on intrinsic bone mechanical properties}

The intrinsic bone tissue mechanical properties, displayed as elastic modulus $\mathrm{E}_{\mathrm{r}}$ and hardness $\mathrm{H}$ and quantified by nanoindentation, are shown in Figure 6. Overall, the SR treatment at the high dose improved the reduced mechanical properties of subchondral trabecular bone induced by MMT. Specifically, compared with $20.43 \pm 3.37 \mathrm{GPa}$ at 3 weeks and $21.12 \pm 3.96$ GPa at 6 weeks for the Sham/NS animals, the elastic modulus was reduced to $15.95 \pm 3.09 \mathrm{GPa}(P<0.01)$ and $14.14 \pm 3.86$ GPa $(P<0.01)$, respectively, for the MMT/NS animals. In contrast, the decreased elastic modulus in the MMT/NS animals was significantly improved by the high-dose SR treatment $(20.00 \pm 3.93 \mathrm{GPa}, P<0.05$ at 3 weeks and $19.07 \pm 4.41 \mathrm{GPa}$, $P<0.01$ at 6 weeks) but not by the SR treatment at the low dose $(17.52 \pm 3.45 \mathrm{GPa}, P>0.05$ at 3 weeks and $16.03 \pm 3.80 \mathrm{GPa}, P>0.05$ at 6 weeks). Hardness was also reduced in the MMT/NS 

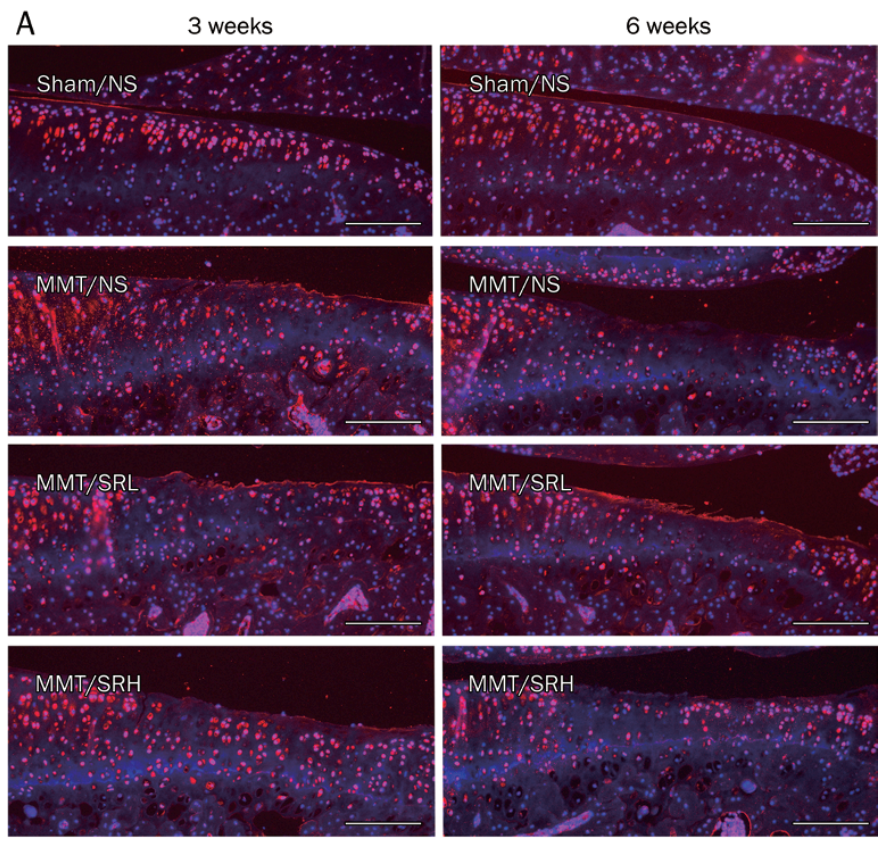

B

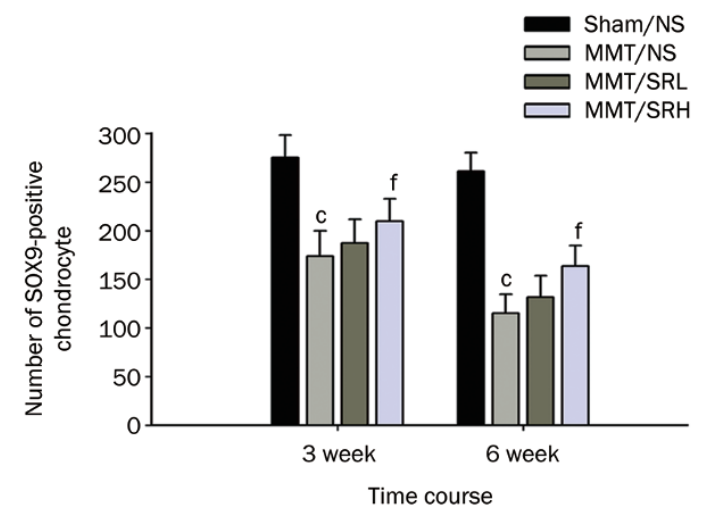

Figure 3. Effect of SR treatment on SOX9 expression in articular chondrocytes. Representative images of SOX9 expression by immunofluorescence. Red indicates SOX9-immunopositive chondrocytes. The images were acquired at $\times 100$ magnification. Bars $=200 \mu \mathrm{m}$. (B) Analysis of the number of SOX9-immunopositive chondrocytes. $n=8$ per group. Mean \pm SD. ${ }^{c} P<0.01$ vs Sham/NS group. ${ }^{f} P<0.01$ vs the MMT/NS group.

animals compared with the Sham/NS animals $(0.74 \pm 0.13$ GPa to $0.91 \pm 0.15 \mathrm{GPa}, P<0.05$ at 3 weeks and $0.69 \pm 0.13 \mathrm{GPa}$ to $0.94 \pm 0.15 \mathrm{GPa}, P<0.01$ at 6 weeks), which was significantly preserved by the SR treatment at the high dose $(0.89 \pm 0.16 \mathrm{GPa}$, $P<0.05$ at 3 weeks and $0.85 \pm 0.12 \mathrm{GPa}, P<0.01$ at 6 weeks) but not by the low dose $(0.82 \pm 0.12 \mathrm{GPa}, P>0.05$ at 3 weeks and $0.78 \pm 0.12 \mathrm{GPa}, P>0.05$ at 6 weeks).

\section{Discussion}

The well-established MMT model was employed in the present study to mimic clinically relevant OA degeneration ${ }^{[33]}$. Consistent with previous results ${ }^{[24,34,35]}$, the present study demonstrated that the MMT operation induced articular cartilage pathology, indicated by cartilage matrix and chondrocyte loss, increased chondrocytes apoptosis and decreased SOX9 expression.

Normal articular chondrocytes express SOX9, a critical transcription factor responsible for the expression of the anabolic genes type II collagen and aggrecan ${ }^{[36]}$. SOX9 plays key roles in cartilage development and maintenance of the chondrocyte phenotype ${ }^{[37]}$. The downregulation of SOX9 is one characteristic change of cartilage degeneration in $\mathrm{OA}^{[38,39]}$. The present findings demonstrated that SR treatment at $1800 \mathrm{mg} \cdot \mathrm{kg}^{-1} \cdot \mathrm{d}^{-1}$ significantly improved SOX9 immunoexpression in the rat MMT OA model. However, whether SR has a direct regulatory effect on the expression of SOX9 in the chondrocytes is not clear.

Bone is a complex tissue, with its overall properties depending not only on quantity but also on quality, characterized by its structure and material composition (mineral and collagen) ${ }^{[40]}$. Therefore, to evaluate the overall properties of subchondral bone, the current study examined the commonly used microarchitecture indices and the potential changes in TMD and $\mathrm{v}_{1}-\mathrm{PO}_{4}{ }^{3-} /$ proline. In addition, nanoindentation, which has emerged as a powerful tool to investigate variations in the mechanical properties of trabecular and cortical bone $^{[11,42]}$, was used to assess the intrinsic mechanical properties (hardness and modulus) of subchondral trabecular bone. The current data demonstrated that the MMT model showed decreased TMD, $\mathrm{v}_{1}-\mathrm{PO}_{4}{ }^{3-} /$ proline and intrinsic mechanical properties of the subchondral trabecular bone; these findings are in accordance with previous results showing that subchondral bone from OA patients displayed a higher turnover rate, decreased mineralisation, elevated collagen content, and weaker mechanical strength ${ }^{[9,43]}$.

Previous studies have revealed that targeting subchondral bone with bone-modifying agents ${ }^{[4]}$, such as bisphosphonates, estrogens, calcitonins, osteoprotegerin, PTH (1-34), have protective effects on subchondral bone and articular cartilage in OA with or without osteoporosis. SR, unlike any other bonemodifying drug, has a dual effect on bone remodeling, being able to stimulate bone formation by osteoblasts, a property shared with bone-forming agents, and inhibit bone resorption by osteoclasts, similar to anti-resorptive agents ${ }^{[19,20]}$. SR has displayed the ability to rebalance bone turnover, improve bone morphology, preserve the degree of mineralization, and restore bone biomechanical properties in in vitro, pre-clinical and clinical studies ${ }^{[19,20,4]}$. The current study demonstrated that oral SR treatment at $1800 \mathrm{mg} \cdot \mathrm{kg}^{-1} \cdot \mathrm{d}^{-1}$ was able to attenuate articular cartilage degeneration in the rat MMT model while effectively preserving the properties of subchondral bone, as indicated by improvements in morphology, microarchitecture indices, TMD, $\mathrm{v}_{1}-\mathrm{PO}_{4}^{3-} /$ proline and intrinsic mechanical properties.

Strontium $\left(\mathrm{Sr}^{2+}\right)$ has a great affinity for bone. $\mathrm{Sr}^{2+}$ is incorporated into crystal lattice of the bone mineral by ionic exchange with bone $\mathrm{Ca}^{2+[45]}$. Thus, the incorporation of $\mathrm{Sr}^{2+}$ by itself may have an effect on the physical properties of bone, correspondingly, which may partly contribute to the change of our indentation results after SR administration. However, in treated 
A
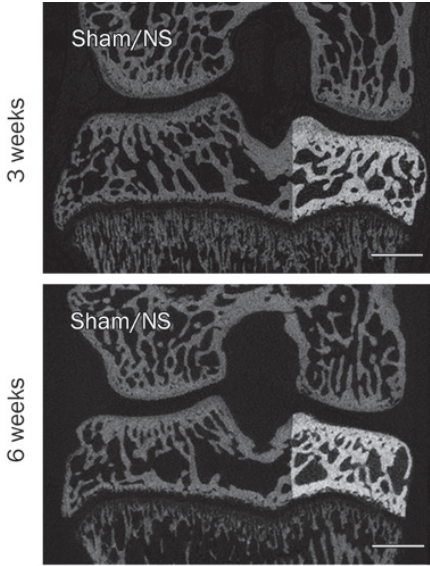
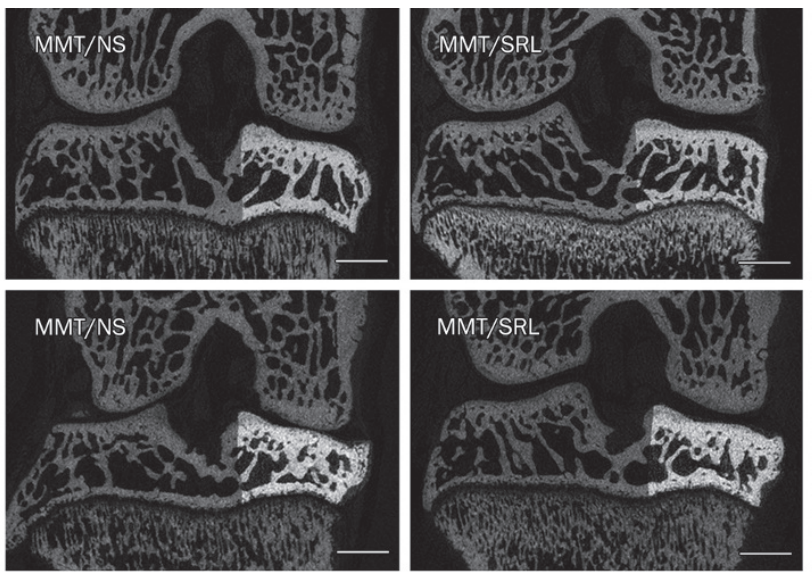
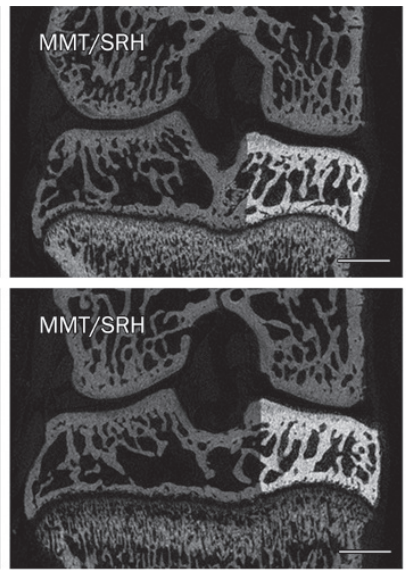

B

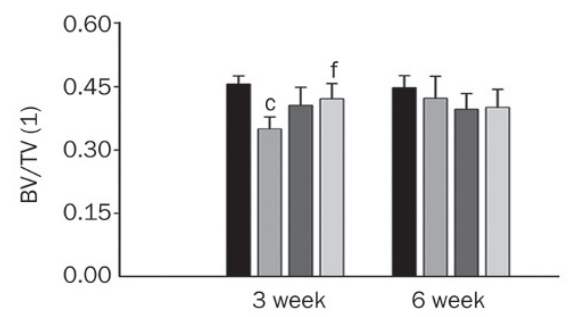

Time course

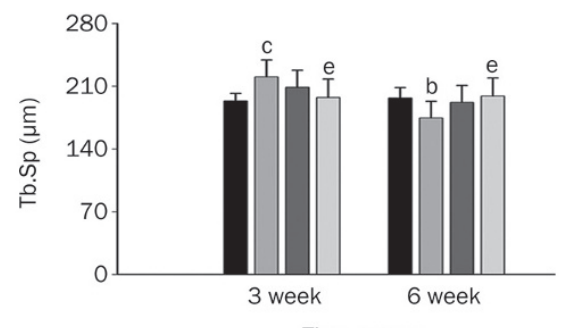

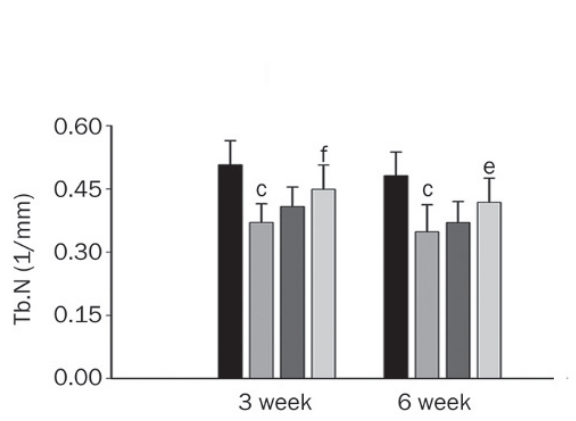

Time course

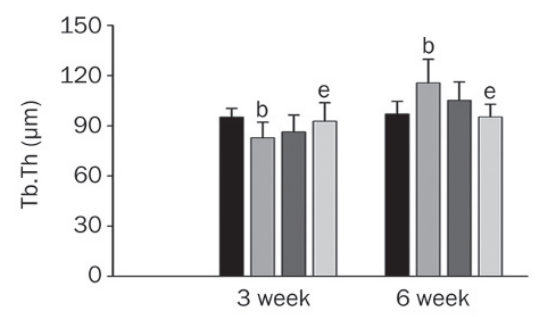

Time course

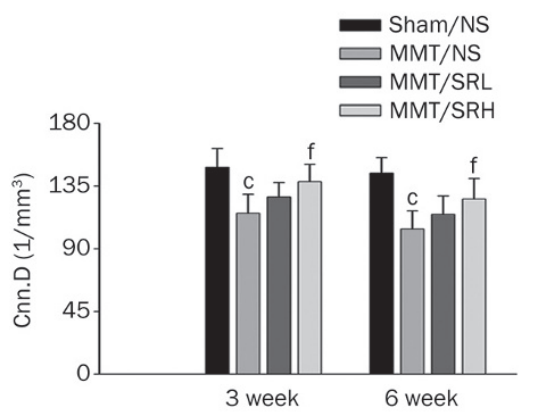

Time course

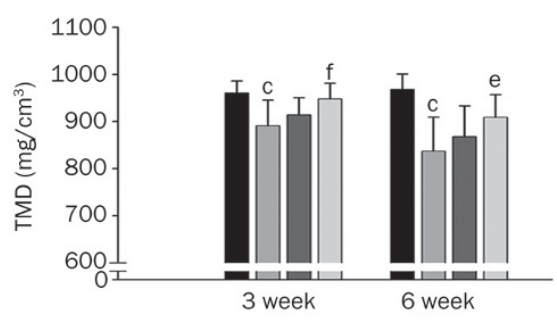

Time course

Figure 4. Effect of SR treatment on subchondral bone microarchitecture and tissue mineral density (TMD). (A) Representative images of the knee obtained using micro-CT scanning. Bars $=1000 \mu \mathrm{m}$. (B) Analysis of subchondral bone microstructure parameters and TMD. $n=8$ per group. Mean \pm SD. ${ }^{\mathrm{b}} P<0.05,{ }^{\mathrm{c}} P<0.01$ vs Sham/NS group. ${ }^{\mathrm{e}} P<0.05,{ }^{\mathrm{f}} P<0.01$ vs MMT/NS group.

animals, even with large doses over a long period of time, the total amount of $\mathrm{Sr}^{2+}$ in bone is always very low as compared with calcium (only a few percent of the bone calcium content), because a theoretical maximum of one calcium atom out of ten can be substituted by a $\mathrm{Sr}^{2+[45]}$. Compared with its effect on osteoblast/osteoclast function, the incorporation of $\mathrm{Sr}^{2+}$ into bone should play a small role in the changes of indentation results.

Earlier studies have shown that SR treatment at a dose of $625 \mathrm{mg} \cdot \mathrm{kg}^{-1} \cdot \mathrm{d}^{-1}$ led to a blood strontium concentration in experimental rats corresponding to that in patients administered the therapeutic dose of $2 \mathrm{~g} / \mathrm{d}$ and demonstrated an improvement in the trabecular and cortical bone tissue architecture, mass, quality and strength in rats with or without osteoporosis $^{[25,46,47]}$. However, the current data demonstrated that the commonly used dose of $625 \mathrm{mg} \cdot \mathrm{kg}^{-1} \cdot \mathrm{d}^{-1}$ showed no statistical improvement in the subchondral bone pathology in the rat MMT model, although tendencies toward improvement were observed. This difference was also observed in previous studies that investigated the effect of other bonemodifying agents and found that a higher dose than that used in osteoporosis treatment may be needed to inhibit joint degeneration ${ }^{[4]}$. One potential explanation for this difference may be that the subchondral bone turnover in osteoarthritis is much higher than that occurring in osteoporosis, which could not be antagonized effectively by the routine dose of bone-modifying agents. Another possibility may be that the disease progression is fast in this MMT model, which leaves a relatively small window for potential pharmaceutical testing; thus, pre-dosing animals to steady state plasma concentrations prior to initiating the MMT surgery may be desirable ${ }^{[33]}$.

The current findings that SR treatment exerts a favorable 

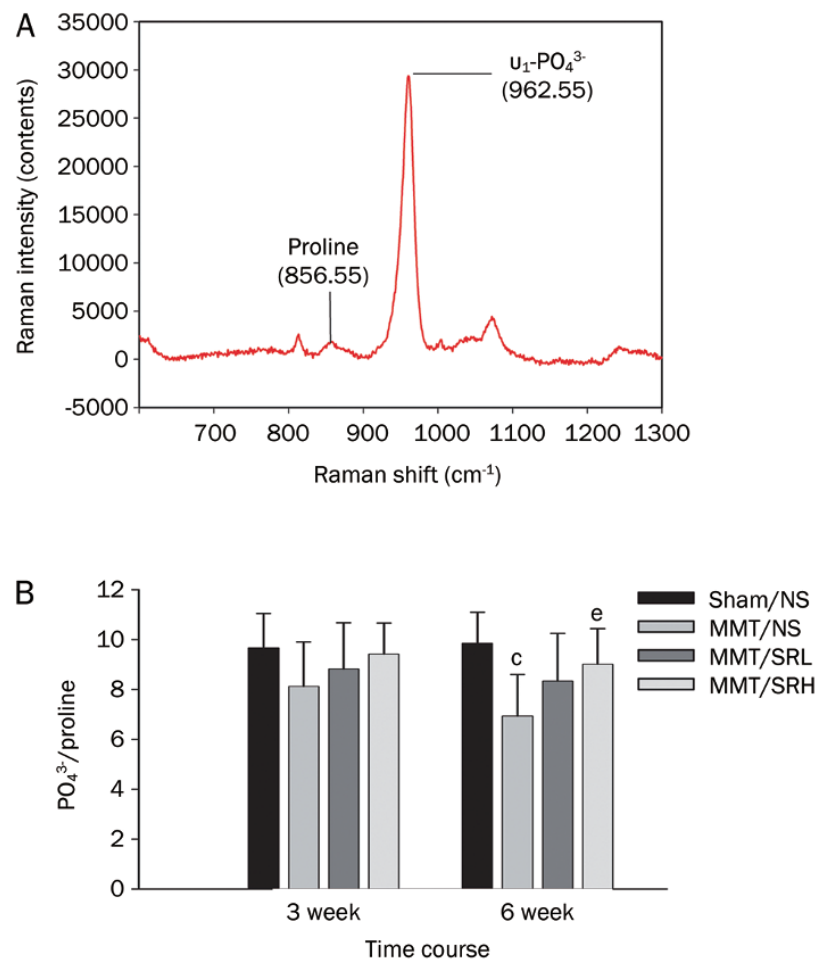

Figure 5. Effect of SR treatment on subchondral bone mineral-to-collagen ratio. A typical Raman spectrum from the subchondral trabecula. The mineral-to-collagen ratio $\left(\mathrm{U}_{1}-\mathrm{PO}_{4}{ }^{3-} /\right.$ proline $)$ was calculated as the raw $\mathrm{U}_{1}$ phosphate peak intensity $\left(\mathrm{U}_{1}-\mathrm{PO}_{4}{ }^{3-}, 962 \mathrm{~cm}^{-1}\right)$ per proline peak intensity $\left(856 \mathrm{~cm}^{-1}\right)$. (B) Analysis of the subchondral bone $\mathrm{U}_{1}-\mathrm{PO}_{4}{ }^{3} /$ proline. $n=8$ per group. Mean \pm SD. ${ }^{c} P<0.01$ compared with the Sham/NS group. ${ }^{\mathrm{e}} P<0.05,{ }^{\mathrm{f}} P<0.01$ vs MMT/NS group.

effect on osteoarthritic cartilage and subchondral bone pathology are supported by findings from several earlier studies. SR was reported to reduce the progression of radiographic features of spinal OA in patients with concurrent osteoporosis and spinal $\mathrm{OA}^{[48]}$ and reduce the urinary level of the type II collagen degradation biomarker CTX-II in postmenopausal women $^{[49]}$ and in postmenopausal women with different clinical levels of $\mathrm{OA}^{[50]}$.

In conclusion, using the rat MMT OA model, the present findings indicate that oral SR treatment could reduce the articular cartilage and subchondral bone pathology and support further evaluation of the therapeutic potential of SR treatment.

\section{Acknowledgements}

This work was supported by grants from the National Natural Science Foundation of China (№ 30700852, 30973038), the Fund for Key Disciplines of Shanghai Municipal Education Commission (J50206) and the Program for the Shanghai Key Laboratory of Orthopedic Implants (Grant № 08DZ2230330). .

\section{Author contribution}

De-gang YU, Hui-feng DING, and Yuan-qing MAO perfor most of the animal and histological experiments. Ming LIU
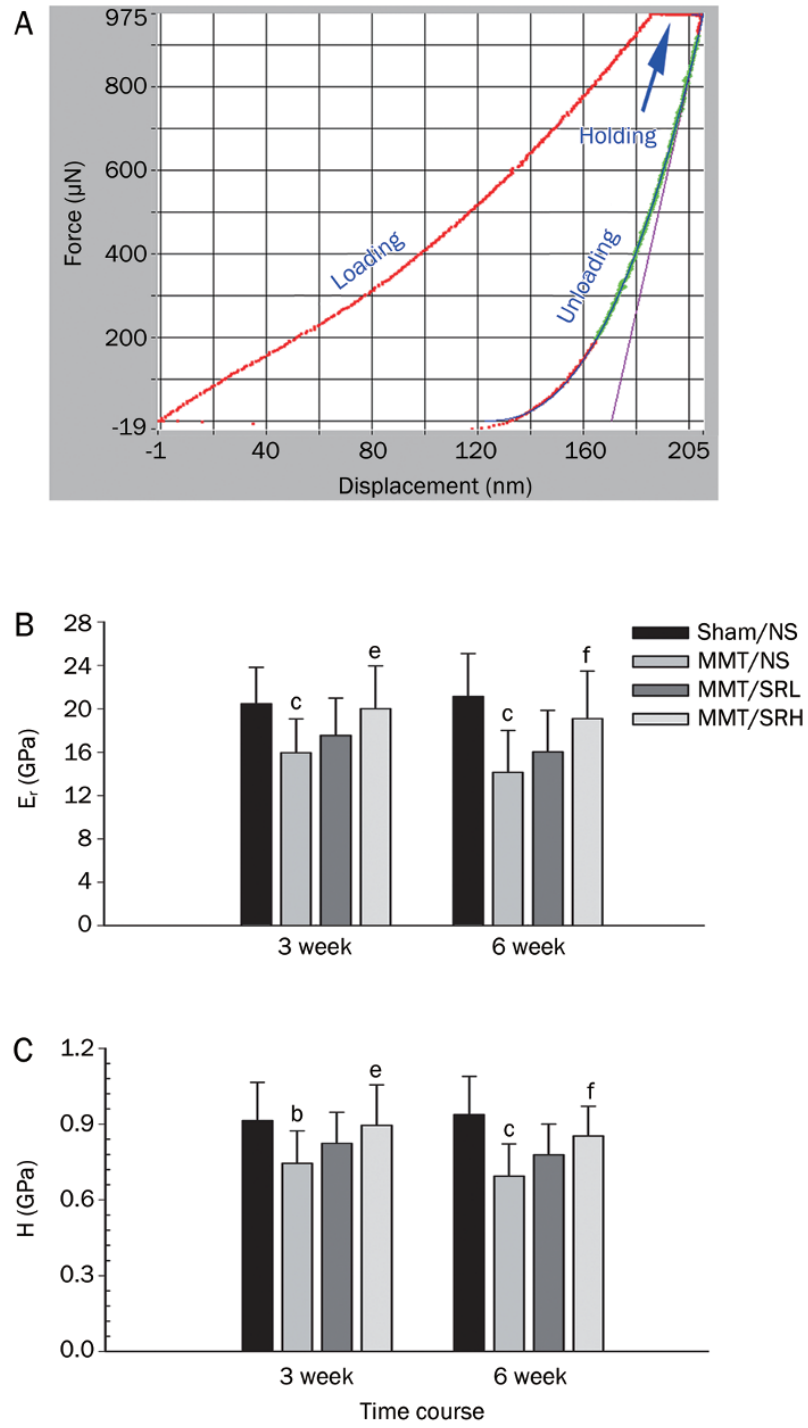

Figure 6. Effect of SR treatment on intrinsic bone mechanical properties. (A) Representative nanoindentation load-displacement curve from the subchondral trabecula. The elastic modulus $\left(E_{r}\right)$ and hardness $(H)$ of the tissue at the point of indention was calculated following the method of Oliver and Pharr. ( $B$ and $C$ ) Analysis of $E_{r}$ and $H$. $n=8$ per group. Mean \pm SD. ${ }^{b} P<0.05,{ }^{c} P<0.01$ vs Sham/NS group. ${ }^{e} P<0.05,{ }^{f} P<0.01$ vs $\mathrm{MMT} / \mathrm{NS}$ group.

and Xin ZHAO performed the micro-CT imaging experiments. Bo YU, Xiao-qing WANG, Yang LI, and Guang-wang LIU performed the Raman and nanoindentation analysis. Shao-bo NIE, Shen LIU, and Zhen-an ZHU conceived the study, designed the experiments, participated in the data analysis and helped draft the manuscript.

\section{References}

1 Holman HR, Lorig KR. Overcoming barriers to successful aging. Selfmanagement of osteoarthritis. West J Med 1997; 167: 265-8.

2 Lane NE, Brandt K, Hawker G, Peeva E, Schreyer E, Tsuji W, et al. OARSI-FDA initiative: defining the disease state of osteoarthritis. Osteoarthritis Cartilage 2011; 19: 478-82. 
3 Kwan Tat S, Lajeunesse D, Pelletier JP, Martel-Pelletier J. Targeting subchondral bone for treating osteoarthritis: what is the evidence? Best Pract Res Clin Rheumatol 2010; 24: 51-70.

4 Castañeda S, Roman-Blas J, Largo R, Herrero-Beaumont G. Subchondral bone as a key target for osteoarthritis treatment. Biochem Pharmacol 2012; 83: 315.

5 Imhof H, Sulzbacher I, Grampp S, Czerny C, Youssefzadeh S, Kainberger $\mathrm{F}$. Subchondral bone and cartilage disease: a rediscovered functional unit. Invest Radiol 2000; 35: 581.

6 Malinin T, Ouellette E. Articular cartilage nutrition is mediated by subchondral bone: a long-term autograft study in baboons. Osteoarthritis Cartilage 2000; 8: 483-91.

7 Karsdal MA, Leeming DJ, Dam EB, Henriksen K, Alexandersen P, Pastoureau $\mathrm{P}$, et al. Should subchondral bone turnover be targeted when treating osteoarthritis? Osteoarthritis Cartilage 2008; 16: 63846.

8 Goldring SR. Role of bone in osteoarthritis pathogenesis. Med Clin North Am 2009; 93: 25-35.

9 Li B, Aspden RM. Composition and mechanical properties of cancellous bone from the femoral head of patients with osteoporosis or osteoarthritis. J Bone Mineral Res 1997; 12: 641-51.

10 Bailey AJ, Mansell JP, Sims TJ, Banse X. Biochemical and mechanical properties of subchondral bone in osteoarthritis. Biorheology 2004; 41: 349-58.

11 Burr DB. Anatomy and physiology of the mineralized tissues: role in the pathogenesis of osteoarthrosis. Osteoarthritis Cartilage 2004; 12: S20-30.

12 Sanchez C, Deberg M, Piccardi N, Msika P, Reginster JYL, Henrotin Y. Subchondral bone osteoblasts induce phenotypic chang es in human osteoarthritic chondrocytes. Osteoarthritis Cartilage 2005; 13 : 988-97.

13 Sanchez C, Deberg MA, Bellahcène A, Castronovo V, Msika P, Delcour $\mathrm{JP}$, et al. Phenotypic characterization of osteoblasts from the sclerotic zones of osteoarthritic subchondral bone. Arthritis Rheum 2008; 58 : 442-55.

14 Chan TF, Couchourel D, Abed É, Delalandre A, Duval N, Lajeunesse D. Elevated Dickkopf-2 levels contribute to the abnormal phenotype of human osteoarthritic osteoblasts. J Bone Mineral Res 2011; 26 : 1399-410.

15 Lajeunesse D, Reboul P. Subchondral bone in osteoarthritis: a biologic link with articular cartilage leading to abnormal remodeling. Curr Opin Rheumatol 2003; 15: 628.

16 Prasadam I, van Gennip S, Friis T, Shi W, Crawford R, Xiao Y. ERK-1/2 and $\mathrm{p} 38$ in the regulation of hypertrophic changes of normal articular cartilage chondrocytes induced by osteoarthritic subchondral osteoblasts. Arthritis Rheum 2010; 62: 1349-60.

17 Goldring MB, Goldring SR. Articular cartilage and subchondral bone in the pathogenesis of osteoarthritis. Ann N Y Acad Sci 2010; 1192: 230-7.

18 Lories RJ, Luyten FP. The bone-cartilage unit in osteoarthritis. Nat Rev Rheumatol 2011; 7: 43-9.

19 Fonseca J. Rebalancing bone turnover in favour of formation with strontium ranelate: implications for bone strength. Rheumatology (Oxford) 2008; 47: iv17-iv19.

20 Hamdy NAT. Strontium ranelate improves bone microarchitecture in osteoporosis. Rheumatology (Oxford) 2009; 48: iv9-iv13.

21 Henrotin Y, Labasse A, Zheng S, Galais P, Tsouderos Y, Crielaard JM, et al. Strontium ranelate increases cartilage matrix formation. J Bone Mineral Res 2001; 16: 299-308.

22 Tat SK, Pelletier JP, Mineau F, Caron J, Martel-Pelletier J. Strontium ranelate inhibits key factors affecting bone remodeling in human os- teoarthritic subchondral bone osteoblasts. Bone 2011; 49: 559-67.

23 Janusz MJ, Bendele AM, Brown KK, Taiwo YO, Hsieh L, Heitmeyer SA. Induction of osteoarthritis in the rat by surgical tear of the meniscus: Inhibition of joint damage by a matrix metalloproteinase inhibitor. Osteoarthritis Cartilage 2002; 10: 785-91.

24 Gerwin N, Bendele AM, Glasson S, Carlson CS. The OARSI histopathology initiative - recommendations for histological assessments of osteoarthritis in the rat [Consensus Development Conference]. Osteoarthritis Cartilage 2010; 18: S24-34.

25 Bain S, Jerome C, Shen V, Dupin-Roger I, Ammann P. Strontium ranelate improves bone strength in ovariectomized rat by positively influencing bone resistance determinants. Osteoporosis Int 2009; 20: 1417-28.

26 Ahmet-Camcioglu N, Okman-Kilic T, Durmus-Altun G, Ekuklu G, Kucuk M. Effects of strontium ranelate, raloxifene and misoprostol on bone mineral density in ovariectomized rats. Eur J Obstet Gynecol Reprod Biol 2009; 147: 192-94.

27 Yu D, Yu B, Mao Y, Zhao X, Wang X, Ding H, et al. Efficacy of zoledronic acid in treatment of teoarthritis is dependent on the disease progression stage in rat medial meniscal tear model. Acta Pharmacol Sin 2012; 33: 924-34.

28 Edwards JR, Nyman JS, Lwin ST, Moore MM, Esparza J, O'Quinn EC, et al. Inhibition of TGF- $\beta$ signaling by 1D11 antibody treatment increases bone mass and quality in vivo. J Bone Mineral Res 2010; 25 : 2419-26.

29 Nyman JS, Makowski AJ, Patil CA, Masui TP, O'Quinn EC, Bi X, et al. Measuring differences in compositional properties of bone tissue by confocal raman spectroscopy. Calcif Tissue Int 2011: 89: 111-22.

30 Lieber CA, Mahadevan-Jansen A. Automated method for subtraction of fluorescence from biological Raman spectra. Appl Spectrosc 2003; 57: 1363-67.

31 Gupta H, Schratter S, Tesch W, Roschger P, Berzlanovich A, Schoeberl $\mathrm{T}$, et al. Two different correlations between nanoindentation modulus and mineral content in the bone-cartilage interface. J Struct Biol 2005; 149: 138-48.

32 Oliver WC, Pharr GM. Improved technique for determining hardness and elastic modulus using load and displacement sensing indentation experiments. J Materials Res 1992; 7: 1564-83.

33 Bendele A. Animal models of osteoarthritis. J Musculoskelet Neuronal Interact 2001; 1: 363-76.

34 Blanco FJ, Guitian R, Vázquez-Martul E, de Toro FJ, Galdo F. Osteoarthritis chondrocytes die by apoptosis: a possible pathway for osteoarthritis pathology. Arthritis Rheumatism 1998; 41: 284-89.

35 Hashimoto M, Nakasa T, Hikata T, Asahara H. Molecular network of cartilage homeostasis and osteoarthritis. Med Res Rev 2008; 28 : 464-81.

36 Salminen H, Vuorio E, Säämänen AM. Expression of Sox9 and type IIA procollagen during attempted repair of articular cartilage damage in a transgenic mouse model of osteoarthritis. Arthritis Rheumatism 2001; 44: 947-55.

37 de Crombrugghe B, Lefebvre V, Nakashima K. Regulatory mechanisms in the pathways of cartilage and bone formation. Curr Opin Cell Biol 2001; 13: 721-8.

38 Appleton CTG, Usmani SE, Bernier SM, Aigner T, Beier F. Transforming growth factor $\alpha$ suppression of articular chondrocyte phenotype and Sox 9 expression in a rat model of osteoarthritis. Arthritis Rheumatism 2007; 56: 3693-705.

39 Tew SR, Clegg PD, Brew CJ, Redmond CM, Hardingham TE. SOX9 transduction of a human chondrocytic cell line identifies novel genes regulated in primary human chondrocytes and in osteoarthritis. Arthritis Res Ther 2007; 9: R107. 
40 Viguet-Carrin S, Garnero P, Delmas P. The role of collagen in bone strength. Osteoporosis Int 2006; 17: 319-36.

41 Ebenstein DM, Pruitt LA. Nanoindentation of biological materials. Nano Today 2006; 1: 26-33.

42 Lewis G, Nyman JS. The use of nanoindentation for characterizing the properties of mineralized hard tissues: State-of-the art review. J Biomed Mater Res B Appl Biomater 2008; 87: 286-301.

43 Coats AM, Zioupos P, Aspden RM. Material properties of subchondral bone from patients with osteoporosis or osteoarthritis by microindentation testing and electron probe microanalysis. Calcif Tissue Int 2003; 73: 66-71.

44 Marie P, Felsenberg D, Brandi M. How strontium ranelate, via opposite effects on bone resorption and formation, prevents osteoporosis. Osteoporosis Int 2011; 22: 1659-67.

45 Dahl S, Allain P, Marie P, Mauras Y, Boivin G, Ammann P, et al. Incorporation and distribution of strontium in bone. Bone 2001; 28 : 446-53.

46 Ammann P, Shen V, Robin B, Mauras Y, Bonjour JP, Rizzoli R. Stron- tium ranelate improves bone resistance by increasing bone mass and improving architecture in intact female rats. J Bone Mineral Res 2004; 19: 2012-20.

47 Ammann P, Badoud I, Barraud S, Dayer R, Rizzoli R. Strontium ranelate treatment improves trabecular and cortical intrinsic bone tissue quality, a determinant of bone strength. J Bone Mineral Res 2007; 22: 1419-25.

48 Bruyere O, Delferriere D, Roux C, Wark JD, Spector T, Devogelaer JP, et al. Effects of strontium ranelate on spinal osteoarthritis progression. Ann Rheum Dis 2008; 67: 335-9.

49 Alexandersen P, Karsdal M, Qvist P, Reginster JY, Christiansen C. Strontium ranelate reduces the urinary level of cartilage degradation biomarker CTX-II in postmenopausal women. Bone 2007; 40: 21822.

50 Alexandersen P, Karsdal M, Byrjalsen I, Christiansen C. Strontium ranelate effect in postmenopausal women with different clinical levels of osteoarthritis. Climacteric 2011; 14: 236-43. 